\title{
$\mathrm{CiSj}$
}

\section{AN INFLUENCE OF PARAMETER VARIATION ON MODEL-BASED EMERGENCY FORECASTIING IN THE PULSE SYSTEM}

\author{
Yury Kolokolov, Anna Monovskaya
}

\author{
JSC "Electrotex" \\ 5, Naygorskoye Shosse, 302020, Orel, Russia, \\ Department of Design and Technology of Electronic Systems \\ State Technical University of Orel, \\ 29, Naygorskoye Shosse, 302020, Orel, Russia \\ tel: 70862 421661, fax: 70862 416684, e-mail: anna@ostu.ru
}

\begin{abstract}
Practical realization of model-based emergency forecasting in pulse systems for the case of multi-parameter variation in a possible wide range associates with the problem of a-priori forming of single-statement information on system dynamics. The fractal diagram is proposed to use for the purpose of system dynamics presentation. This diagram allows one-to-one correspondence between the both, parameter and phase,spaces establishing by line up stages.
\end{abstract}

Keywords: Pulse energy conversion system, Bifurcation analysis, Dynamics model-based identification and forecasting, Dynamics fractal regularities.

\section{INTRODUCTION}

An application of pulse energy conversion systems (PECS) is one of promising ways to be used for the improvement of an energy conversion process efficiency. According to the investigations [1-4], in the case of possible wide range parameter variations the PECS dynamics is characterized as complicated and multivariated, that stipulate the necessity of emergency forecasting based on realtime PECS dynamics identification.

PECS belong to the class of essentially nonlinear complex dynamic systems with the variable structure of their mathematical models. The most comprehensive information about the same class system dynamics is represented by means of bifurcation and parameter diagram building [4-6]. That is why, the bifurcation approach to modelbased real-time PECS dynamics identification and forecasting was proposed [7-8]. The approach comprises the following steps:

$\checkmark$ a-priori information forming by model-based parameter diagram building on a possible system dynamics within investigated parameter range;

$\checkmark$ a-priori information analysis by parameter space partition into "dangerous" and "non-dangerous" domains for different system stale states;

$\checkmark$ PECS dynamics identification for the particular parameter vector value and dynamics forecasting (including emergencies) regarding the bifurcation boundaries of the parameter diagram. The problem situation of the bifurcation approach practical realization of emergency forecasting in the case of the PECS parameter possible wide range is considered in the paper. Also, one of possible problem decisions on the basis of PECS dynamics fractal regularity use is proposed.

\section{PROBLEM SITUATION}

Let $m$-type of a system motion will be referred to a stable state with the period $T=m a$, then the type of motion $m=1$ will be corresponded to the fundamental mode with the frequency $f_{0}=1 / a$. The stability loss of $m=1$ motion type will be regarded as an emergency. The variation of PESC parameters (P), as a temperature trend, random parameter values fluctuations, system degradation, etc., will be considered as a reason of system dynamics (system motion type) evolution. That is why, deviation of the state vector $(\mathbf{X})$ trajectory from the previous motion trajectory will take place. In such way, by analogy with the Cauchy problems, it will be necessary to solve the inverse dynamics identification problem. The comparative characteristics of the inverse and direct dynamics identification problems are given in Table 1. 
An absence of one-to-one correspondence between the $\mathbf{X}$ - and $\mathbf{P}$-spaces is the main complexity of this problem solving. On the one hand, there are domains in the system parameter space with more than only one possible motion - multi-motion domains (for instance, in Fig. 1b: an intersection of the motion domains $D_{1} \cap D_{2}=D_{\text {multi }(1,2)}$ to which the multi-motions type corresponds in Fig. 1a (in the middle)). In this case, the set of different motion type phase trajectories corresponds to every one parameter vector of the multi-motion domain $\mathrm{P}_{\text {multy }}$ (for instance, multi-motion type in Fig. 1a (in the middle) consists of $m_{1^{-}}$and $m_{2}$-types of motions in Fig. 1a (in the left) and 1a (in the right) accordingly). Therefore dynamics evolution versions may be different (for instance, several possible dynamics evolution versions, that were projected from parameter diagram domain $\mathrm{D}_{\text {multi }}$ to $\left(\boldsymbol{p}_{1}, \boldsymbol{m}\right)$ space are shown in Fig. 1c - so called branching patterns [9]).

Table 1. Dynamics identification problems

\begin{tabular}{|c|c|}
\hline \multicolumn{2}{|c|}{$\begin{array}{l}\text { Dynamics identification problems } \\
\text { in the }(P, X, m, t) \text {-space }\end{array}$} \\
\hline direct & inverse \\
\hline $\begin{array}{l}\text { Specific reason - uncertain } \\
\text { consequence }\end{array}$ & $\begin{array}{l}\text { Specific consequence - } \\
\text { uncertain reason }\end{array}$ \\
\hline $\begin{array}{l}\text { What will set the system } \\
\text { motion type, if parameter } \\
\text { vector is changing? }\end{array}$ & $\begin{array}{l}\text { Why system dynamics } \\
\text { (parameter vector or (and) } \\
\text { motion type) has changed? }\end{array}$ \\
\hline Specific parameter vector & Uncertain parameter vector \\
\hline $\mathbf{P}=$ var, $\mathbf{X}, \boldsymbol{m} \rightarrow ?$ & $\mathbf{X}=$ var, $\mathbf{P}, \boldsymbol{m} \rightarrow ?$ \\
\hline
\end{tabular}

It means, that both, present dynamics identification and forecasting, regarding the bifurcation boundaries of the parameter diagram need in information not only on $(\boldsymbol{P}, \boldsymbol{m})$-space dynamics, but also on $(\boldsymbol{X}, \boldsymbol{m})$-space dynamics too. On the other hand, the set of parameter vectors with corresponding motion types is possible to confront to the present phase trajectory at different time moments $t$ (for instance, Fig. 1f). Moreover, the mutual disposition of parameter vectors and corresponding phase trajectories (consequently phase vectors and fixed points) may be modified in parameter and phase spaces. For instance, comparative vector mutual dispositions in $(\boldsymbol{P}, \boldsymbol{m})$ and $(\boldsymbol{X}, \boldsymbol{m})$-spaces are shown in Fig. 1d,e. As a result, the uncertainty of the PECS state identification in the multi-dimensional space $(\mathbf{P}, \mathbf{X}, \boldsymbol{m}, \boldsymbol{t})$ is formed.
Complication of a bifurcation boundary pattern leads to a number of dynamics evolution version increasing. Then a number of different consequences of these versions between motions are increasing too. (for instance, in Fig.1g is shown two different scenarios between two system states: 1-4-5-6, 1-36). Nevertheless, one can pick out the typical PECS dynamics evolution cases, that are illustrated in Fig.1g. Let the parameter diagram domain $D_{1}$ contains fundamental mode ( $m_{l}$-type of motion under control). According to mentioned above, this mode changing is regarded as emergency, then there are following cases:

$\checkmark$ the parameter vector of the system is changing only within one-type motion domain. In this case either $m_{1}=$ const (for instance, transition $1 \rightarrow 2$ within only the fundamental motion domain as absence of an emergency), or $m_{1} \neq$ const (for instance, transition $3 \rightarrow 7$ beyond fundamental mode domain as an emergency);

$\checkmark \quad m$ type of motion changes only within multi-type motion domain (for instance, transition-loop $4 \rightarrow 5$ with $m_{1}-t o-m_{3}$ type of motion changes as an emergency);

$\checkmark$ the parameter vector of the system $\mathbf{P}$ are changing as well as the motion type $m$ (for instance, transitions $1 \rightarrow 3 \quad\left(m_{1}-t o-m_{2}, P=v a r\right)$, $1 \rightarrow 4\left(m_{1}=\right.$ const $\cup m_{1}-t o-m_{2}, \mathrm{P}=$ var $), 4 \rightarrow 6$ $\left(m_{3}=\right.$ const $\left.\cup m_{1}-t o-m_{2} \cup m_{1}-t o-m_{3}, \mathrm{P}=\mathrm{var}\right)$, $6 \rightarrow 3\left(m_{2}=\right.$ const $\cup m_{3}-t o-m_{2}, \mathrm{P}=$ var $)-$ as emergencies).

It is necessary to note, that every case contains the possibility of an emergency. A classification of the described transitions is given in Table2. One can see, that one to one correspondence between the both, parameter and phase, spaces may be achieved through by line up stages of system dynamics identification. A-priori information on PX-plane of $(\mathbf{P}, \mathbf{X}, \boldsymbol{m}, \boldsymbol{t})$-space will be formed at the first stage, since it is necessary to safe the fundamental motion state in the system ( $m_{1}$-type of motion).

Table 2. One to one correspondence classification

\begin{tabular}{|l|l|l|l|}
\hline Cases & Variations & Constancies & $\begin{array}{c}\text { One to one } \\
\text { correspondence }\end{array}$ \\
\hline $\begin{array}{l}1 \rightarrow 2, \\
3 \rightarrow 7\end{array}$ & $\mathbf{P}, \mathbf{X}$ & $m$ & $\mathbf{X} \leftrightarrow \mathbf{P}$ \\
\hline $4 \rightarrow 5$ & $m, \mathbf{X}$ & $\mathbf{P}$ & $\mathbf{X} \leftrightarrow m$ \\
\hline $\begin{array}{l}1 \rightarrow 3, \\
1 \rightarrow 4, \\
4 \rightarrow 6, \\
6 \rightarrow 3\end{array}$ & $\mathbf{P}, m, \mathbf{X}$ & - & \\
\hline
\end{tabular}




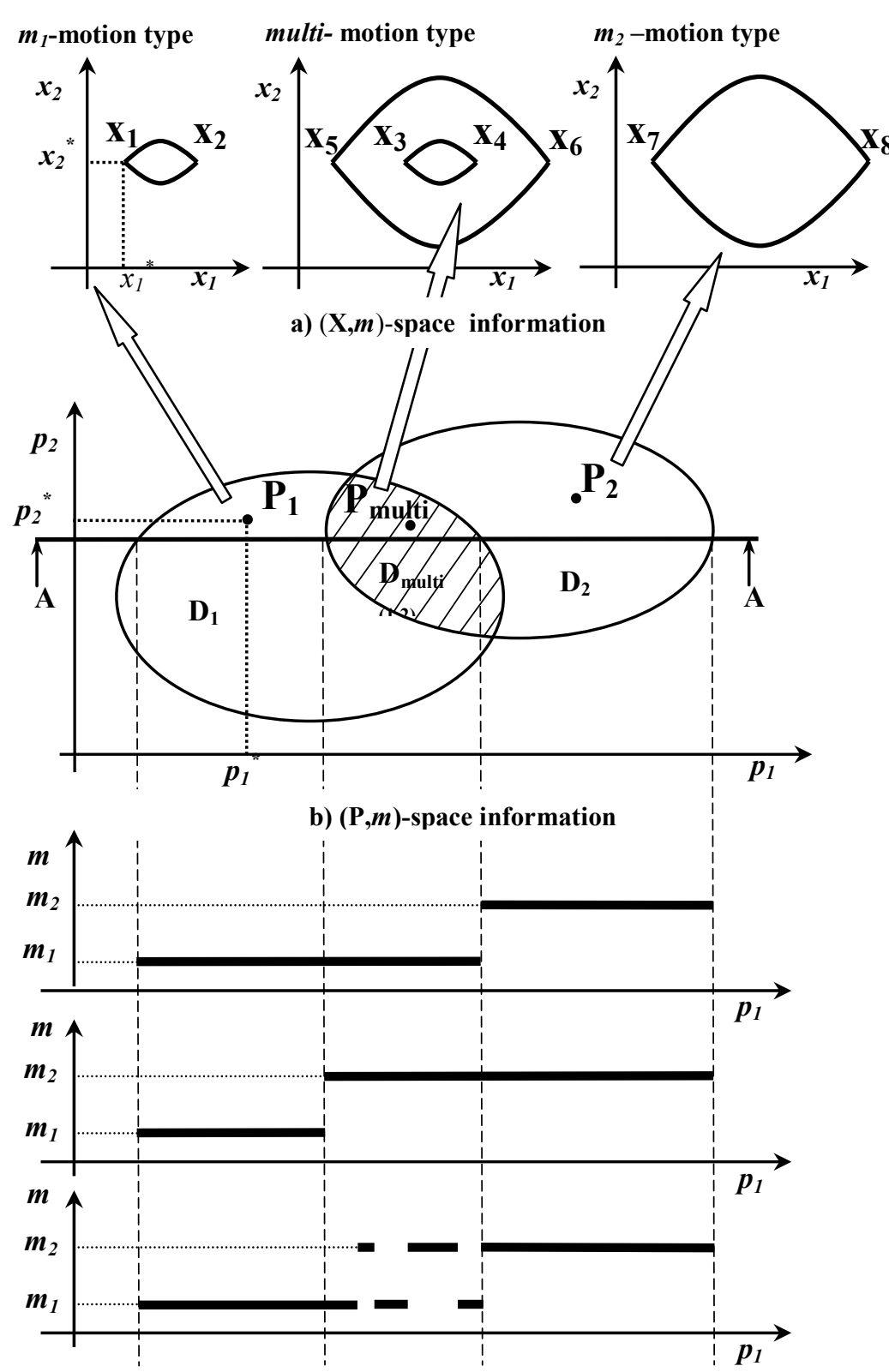

c) $\left(m, p_{1}\right)$-space information

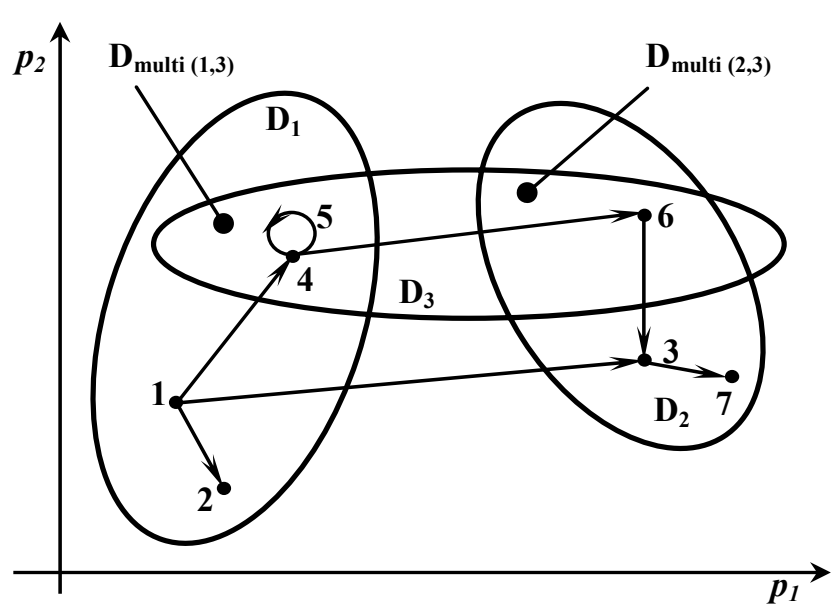

g) $(\mathbf{P}, \boldsymbol{m})$-subspace information

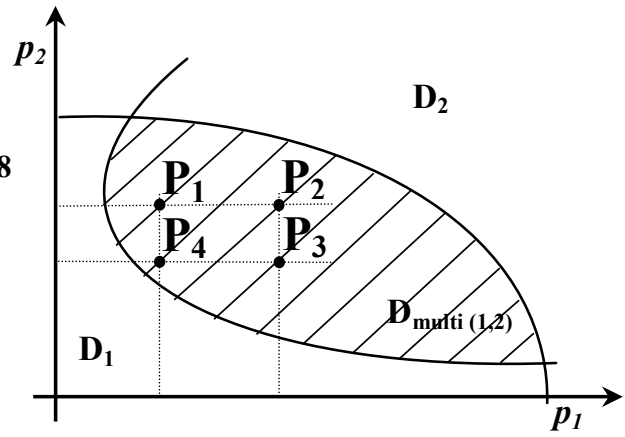

d) $(\mathbf{P}, m)$-space parameter vector mutual disposition

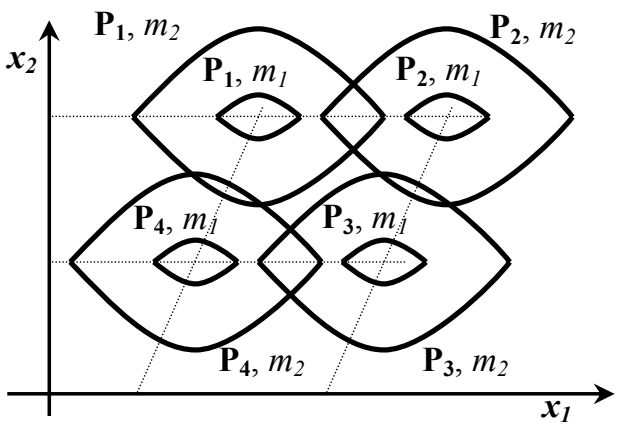

e) $(X, m)$-space phase trajectory mutual disposition

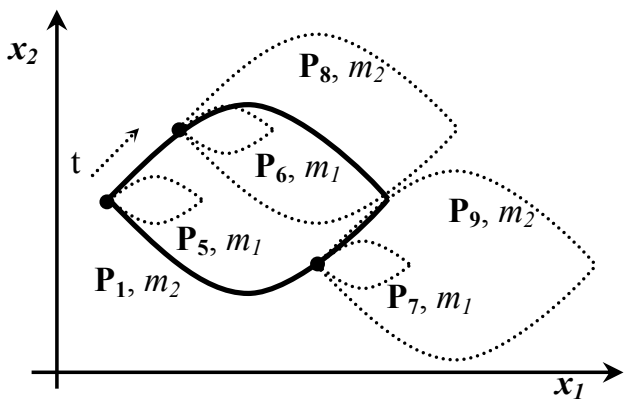

f) dynamics evolution opportunity from the present phase trajectory

$\mathrm{D}_{1}: \mathbf{1} \rightarrow \mathbf{2}$

$\boldsymbol{m}_{1}=$ const, $\mathbf{P}=$ var ;

$D_{2}: 3 \rightarrow 7$

$\boldsymbol{m}_{2}=$ const, $\mathbf{P}=$ var ;

$\mathrm{D}_{1} \rightarrow \mathrm{D}_{2}: \mathbf{1} \rightarrow \mathbf{3}$

$\boldsymbol{m}=$ var, $\mathbf{P}=$ var ;

$D_{1} \rightarrow D_{\text {multi (1,3) }}: \quad 1 \rightarrow 4$

$\boldsymbol{m}_{1}=$ const $\cup \boldsymbol{m}=\mathrm{var}, \mathbf{P}=\mathrm{var}$;

$D_{\text {multi }(1,3)}: \mathbf{4} \rightarrow \mathbf{5}$

$\boldsymbol{m}=$ var, $\mathbf{P}=$ const ;

$D_{\text {multi }(1,3)} \rightarrow D_{\text {multi }(2,3)}: \quad 4 \rightarrow 6$

$\boldsymbol{m}_{3}=$ const $\cup \boldsymbol{m}=$ var, $\mathbf{P}=$ var ;

$D_{\text {multi }(2,3)} \rightarrow D_{2}: 6 \rightarrow 3$

$\boldsymbol{m}_{2}=$ const $\cup \boldsymbol{m}=$ var, $\mathbf{P}=$ var ; 


\section{FRACTAL DIAGRAM}

An approach to practical realization of both, model-based on-line dynamics identification and emergency forecasting, suggesting in the paper, is considered for one of the simplest PECS example (the synchronous buck voltage converter with the second kind pulse-width modulation and proportional regularity low). Because it is sometime helpful to consider small prototype physical systems where fundamental concepts can be more clearly apprehended [10-12]. The equivalent circuit of the buck voltage converter is shown in Fig.2.

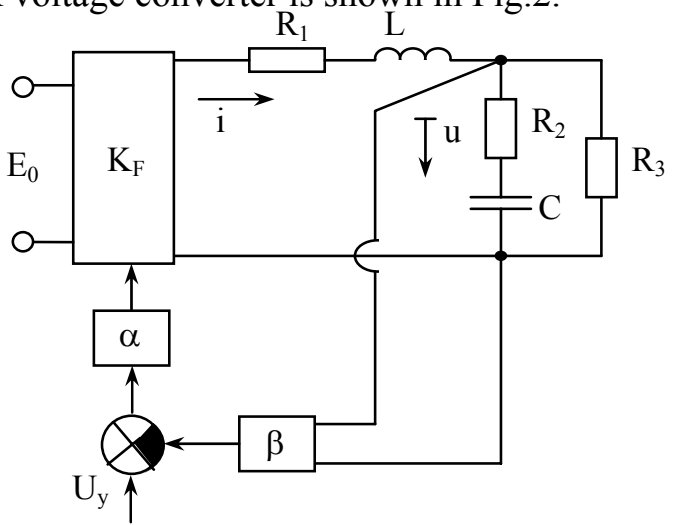

Fig. 2 - Equivalent circuit of the synchronous buck voltage converter

The resulting simplified mathematical model represents the system of two differential equations with a discontinuous right-hand side and a scalar commutation function of a state vector at time. The state vector $\mathbf{X}$ includes the power source current (i) and load voltage $(u)$. The parameter vector $\mathbf{P}$, use for fractal diagram building, includes the error signal amplification coefficient of the voltage regulator $(\alpha)$ and load resistance $\left(R_{3}\right)$

The mathematical model of the system (in Fig. 2) is represented in the terms of shift mapping [4] for the purpose of $\mathbf{P X}$-plane building. In this case, the PECS dynamics from $(\mathbf{P}, \mathbf{X}, \boldsymbol{m}, \boldsymbol{t})$-space is projected into $(\mathbf{P}, \mathbf{X}, \boldsymbol{m})$-space. Let us consider the bifurcation diagram building (in Fig. 3a) in (u,i)-phase space (in Fig. 3b). The obtained geometrical construction presents fractal structure of $1-2-4-\ldots$ scenario with clearly predominate axis of $m=1$ type of motion (in Fig. 3b). The direction of the $\alpha$-axis confronts direction, that was formed by fixed points of mapping of $m=1$ type of motion within an interval of $\alpha$-parameter value changing. Analogous building for $R_{3}$-parameter value interval changing composes the fractal row, as a sequence of topologically equal structures with insignificant dimension modifications (fig. 3c). The $R_{3}$-parameter axis direction confronts the direction of fractal row moving. The obtained geometrical constructions (in Fig.1b,c) will be denoted fractal diagrams. An each point of the fractal diagram presents a fixed point of mapping $(u, i)$ for a parameter vector $\left(R_{3}, \alpha\right)$.

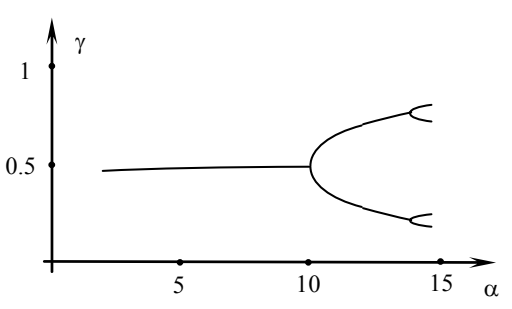

a) bifurcation diagram, $\mathbf{R}_{\mathbf{3}}=\mathbf{1 0}$, $\gamma$ - relative duration of the pulse

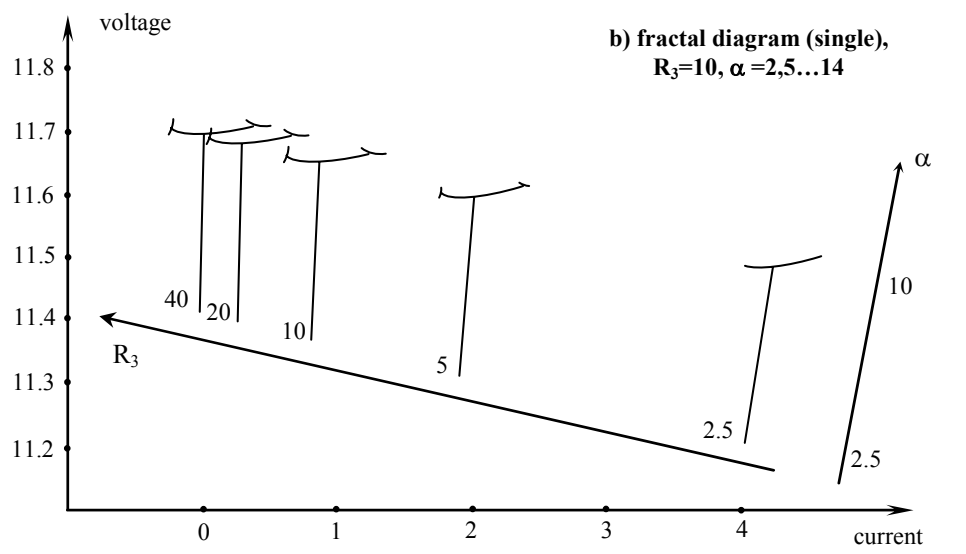

c) b) fractal diagram (row), $R_{3} \in\{2,5 ; 5 ; 10 ; 20 ; 40\}, \alpha=2,5 \ldots 14$

Fig. 3 - Dynamics fractal properties

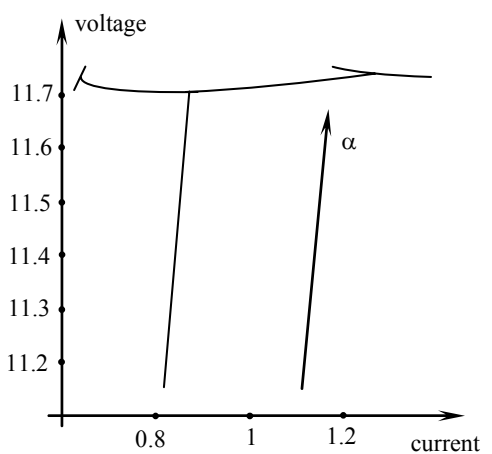

fractal diagram (single) 
Thus, one-to-one correspondence between the both, state $(\mathbf{X})$ and parameter $(\mathbf{P})$, vectors for the specific type of motion (the first dynamics identification stage: $\mathbf{X} \leftrightarrow \mathbf{P}, m=$ const, Table 2.) is a supplementary information property of the fractal diagram. Moreover, phase trajectories of each motion type possesses of its own characteristic fractal structure. Within one-type motion domain phase trajectories are either not changed practically (for instance, for $m=1$ ), or changed by similar mode. There is a common trend that with $m$ increasing a radius of a phase trajectory is also increasing. Hence, it is possible to realize the second dynamics identification stage: one-to-one correspondence between the state vector $\mathbf{X}$ and $m$-type of a motion for the specific parameter vector $\mathbf{P}(\mathbf{X} \leftrightarrow m, \mathbf{P}=$ const, Table 2.). Therefore, the fractal presentation of the PECS dynamics information allows emergency forecasting for the case of a wide range of parameter variation by means of the fundamental motion control: the state space $\mathbf{X}$ changes confront to those of parameter variations.

\section{MULTI-DIMENTIONAL PARAMETER VARIATION IDENTIFICATION}

The following parameter values of the system (in Fig. 2) were used for numerical calculations:
$\mathrm{R}_{1}=0.1 \Omega, \quad \mathrm{R}_{2}=0.1 \Omega, \quad \mathrm{L}=1 \mathrm{e}-4 \mathrm{H}, \quad \mathrm{C}=1 \mathrm{e}-5 \mathrm{~F} . \quad$ The parameters $\alpha \in[2,10]$ и $\mathrm{R}_{3} \in[2,50] \Omega$ were varied and used for fractal diagram building of $1-2-4-\ldots$ dynamics evolution scenario. In this case, a-priori fractal diagram information is quite enough for emergency forecasting algorithm forming according to the following rules:

$\checkmark$ while $m=1$ type of motion maintains in the present system dynamics, the pulse value range of one phase variable $(\Delta i)$ is not practically changed. Therefore, the present parameter vector is one-to-one identified by the present state vector within $m=1$ type motion domain. Thus, $\Delta i$-value will be considered as $m=1$ type of motion identification criterion proceeding from the phase trajectory fractal properties;

$\checkmark \quad$ when another phase variable $(u)$ will be equal to the bifurcation value $u_{1-2}$, then $m=1$ type of motion changes into $m=2$ one. That is why, the $u_{1-2}$-values of the fractal diagram will be considered as the emergency identification criteria within 1-2-4-... dynamics evolution scenario.

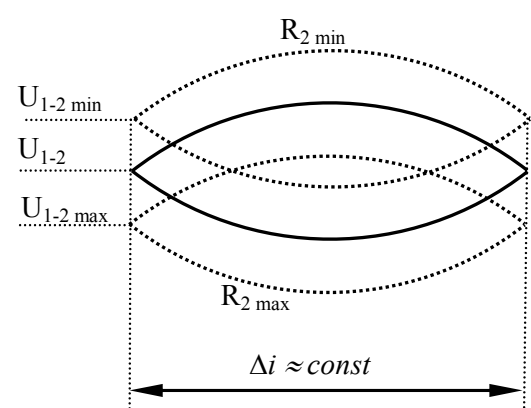

a) $\mathbf{R}_{2}$ - variation

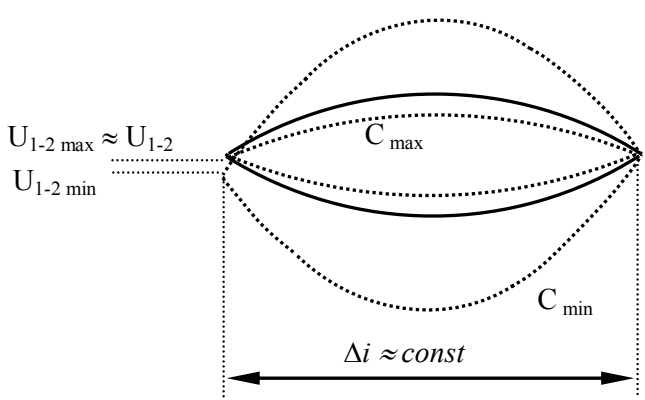

c) C - variation

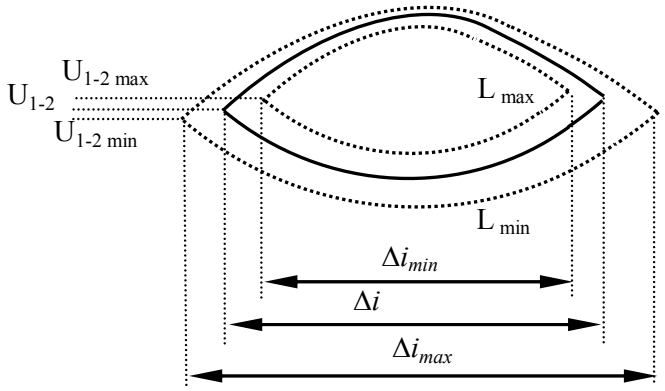

b) L - variation

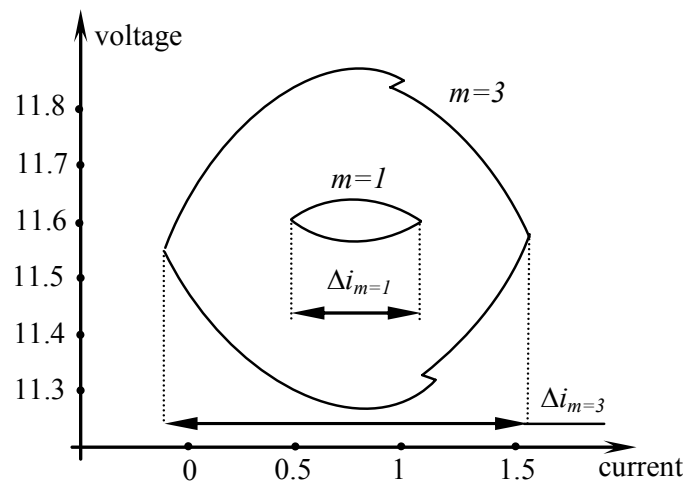

d) bifurcation scenario changing

Fig.4 - Identification criteria

Let us assume, that 3-dimentional parameter variation will be possible, stipulated by the scheme element degradation, as an example. In this case, the estimation of admissible value range of the 
following parameters $\left\{\mathrm{R}_{1}, \mathrm{R}_{2}, \mathrm{~L}, \mathrm{C}\right\}$ are presented in Table 3. One can illustrate, that a-priori 2dementional parameter variation information uncertainty arises. It is exposed by phase trajectory topological transformations, including transformations of identification criterion values $(\Delta i$ and $\left.u_{1-2}\right)$. For instance, the phase trajectory topologically transformations for $\mathrm{R}_{2}, \mathrm{~L}, \mathrm{C}$ parameters is shown in Fig.4 a, b, c accordingly. Nevertheless, one can determine the new identification criterion value range and these value deviations in accordance with the identification criterion values of the fractal diagram (see in Table 3.). One can illustrate, that these deviations are considerably smaller than deviations, revealed in the system dynamics in the case of bifurcation scenario changing. For instance, if $m=1$ type of motion of 1 2-4-.. dynamics evolution scenario changes into $m=3$ type of motion of 3-6-... dynamics evolution scenario, then the identification criterion value $\Delta i$ changes nor less than for $+180 \%$. This example is shown in Fig. 4d.

Table 3. Identification criteria for 1,2,3-dimentional parameter variation

\begin{tabular}{|c|c|c|c|c|c|c|}
\hline \multicolumn{3}{|c|}{ Possible value range of parameter variations } & \multicolumn{2}{|c|}{$\begin{array}{c}\text { Identification criterion of } \\
m=1 \text { type of motion }\end{array}$} & \multicolumn{2}{|c|}{$\begin{array}{c}\text { Type of motion change } \\
\text { identification criterion in the case } \\
\text { of } m=1 \text { to } m=2 \text { changing }\end{array}$} \\
\hline \multicolumn{2}{|c|}{$\begin{array}{c}\text { For fractal diagram } \\
\text { building }\end{array}$} & $\begin{array}{l}\text { Stipulated by scheme } \\
\text { element degradation }\end{array}$ & By value & $\begin{array}{l}\text { Deviation in } \\
\text { accordance } \\
\text { with } \Delta i, \%\end{array}$ & By value & $\begin{array}{c}\text { Deviation in } \\
\text { accordance with } \\
u_{1-2}, \%\end{array}$ \\
\hline \multirow{6}{*}{$\alpha=2 \ldots 10$} & \multirow{6}{*}{$\mathrm{R}_{3}=2 \ldots 50 \Omega$} & - & \multirow{4}{*}{$\Delta i$} & \multirow{4}{*}{ about 0} & \multirow{3}{*}{$\mathrm{u}_{1-2}$} & \multirow{3}{*}{ about 0} \\
\hline & & - & & & & \\
\hline & & $\mathrm{R}_{1}=0,08 \ldots 0,12 \Omega$ & & & & \\
\hline & & $\mathrm{R}_{2}=0,08 \ldots 0,12 \Omega$ & & & $\mathrm{u}_{1-2 \max }$ & $-0,3$ \\
\hline & & $\mathrm{L}=0,8 \ldots 1,2 \mathrm{e}-4 \mathrm{H}$ & $\Delta i_{\min } \ldots \Delta i_{\max }$ & $-20 \ldots+25$ & \multirow{2}{*}{$\mathrm{u}_{1-2 \min }$} & \multirow{2}{*}{$-0,1$} \\
\hline & & $\mathrm{C}=0,4 \ldots 1,6 \mathrm{e}-5 \mathrm{~F}$ & $\Delta i$ & about 0 & & \\
\hline
\end{tabular}

\section{CONCLUSION}

So, the fractal diagram presents 2-dimentional fractal geometrical construction, projecting from $(\mathbf{P}, \mathbf{X}, \boldsymbol{m}, \boldsymbol{t})$-space, which gives the opportunity to statement one-to-one correspondence between spaces of $(\mathbf{P}, \mathbf{X}, \boldsymbol{m}, \boldsymbol{t})$-space. It is possible to pick out fractal structure units, containing sufficient information for the model-based on-line dynamics identification and emergency forecasting practical realization, as well as to estimate evolution directions and boundary existence for these units.

On the basis of the information obtained on PECS dynamics fractal regularities is supposed to form the specific dynamics identification algorithms. It is necessary to denote, that these algorithms are hardly formalized, so characteristic system motions as well as fractal dynamics properties are not known without a priori system dynamics information.

\section{REFERENCES}

[1] S.Banerjee. P.Ranjan. C.Grebogi. Bifurcations in two-dimensional piecewise smooth maps, IEEE Transactions on Circuits and Systems - Theory and
Applications in Switching Circuits 47(5) (2000). p.633-643.

[2] G.Belov. Study of oscillations in the pulse voltage stabilizer near the stability boundary, Electrichestvo 9 (1990). p.44-51.

[3] M.di Bernardo. F.Vasca. Discrete-time maps for the analysis of bifurcations and chaos in DC/DC converters, IEEE Transactions on Circuits and Systems 2(47) (2000). p. 130-142.

[4] Yu.Kolokolov. S.Koschinsky. The regularities of the development of the nonlinear dynamics of the control systems with pulse-width modulation. Proceedings of the "International Conference on Neural Networks and Artificial Intelligence (ICNNAI'99)", Brest, Belarus October 1999, pp. 8592.

[5] M.di Bernardo. Controlling switching systems: A bifurcation approach. Proceedings of the "IEEE International Symposium on Circuits and Systems (ISCAS'2000)", Geneva, Switzerland 2000, vol. II. p.377-378.

[6] A.Nayfeh. A.Harb. C.Chin. A.Hamdan. L.Mili. Application of bifurcation theory to sub- 
synchronous resonance in power systems, Int. J. Bifurcation and Chaos 8(1) (1998). p. 157-172.

[7] Yu. Kolokolov. S.Koschinsky. K.Adjallah. The mathematical problems of forecasting adequacy of emergency situations in the dynamics of the pulse energy conversion systems when using bifurcation approach. Proceeding of the "Second International Conf. on Mathematical methods in Reliability (MMR'2000)", Bordeaux, France 4-7July 2000, pp.603-606.

[8] Yu.Kolokolov. S.Koschinsky. K.Adjallah. Bifurcation approach to condition monitoring: application to pulse energy conversion systems, OPSEARCH 39(1) (2002). p. 1-13.

[9] V.Baushev. Zh.Zhusubaliev. Deterministic operating conditions of a voltage stabilizer with pulse-width modulation control, Electrichestvo 8 (1992). p. 47-53.

[10] Y.Ueda. H.Stewart. R.Abraham. Nonlinear resonance in basin portraits of two coupled swings under periodic forcing, Int. J. Bifurcation and Chaos 8(6) (1998). p. 1183-1197.

[11] L.Malesani. P.Mattavelli. S.Buso. Robust deadbeat current control for PWM rectifies and active filters, IEEE Trans. Ind. Applicat. 35(3 May/June) (1999). p.613-620.

[12] J.Chen. K.Chau. C.Chan. Choose in voltagemode controlled DC drive systems, Int. J. Electron 86(7) (1999). p. 857-874.

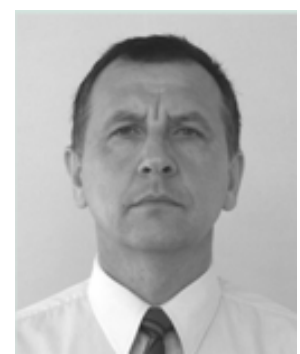

Yury Kolokolov received the M.S. degree in engineering from the Politechnical Institute of Tomsk in June 1972. He defended the thesis for Doctor's of Technical Sciences Degree "Automation of Electromechanical Energy Conversion Processes of D.C. Railway Trains" (State Institute of Automatic Control Systems (ACS)and Radioelectronics, Tomsk, Siberia, Russia) in June 1990. He is currently Professor, Head of Department of Design and Technology of Electronics and Computer Systems, OrelSTU, Russia. His research interests include control and nonlinear dynamics theories and complex electromechanical system study and design.

Anna Monovskaya received the M.S. degree in engineering from the State Technical University of Orel (OrelSTU, Orel, Russia) in June 2001. She is currently working toward the Ph.D. degree in Control Theory at OrelSTU and UTT (Technical University of Troyes, Troyes,

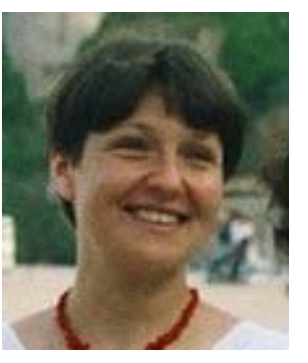

France). Her research interests include control and nonlinear dynamics theories. 